\title{
Association of statin pretreatment with collateral circulation and final infarct volume in acute ischemic stroke patients: A meta-analysis
}

Konark Malhotra $^{\mathrm{a}}$, Apostolos Safouris ${ }^{\mathrm{b}, \mathrm{c}}$, Nitin Goyal ${ }^{\mathrm{d}}$, Adam Arthur ${ }^{\mathrm{e}}$, David S. Liebeskind ${ }^{\mathrm{f}}$, Aristeidis H. Katsanos $^{\mathrm{b}, g}$, João Sargento-Freitas ${ }^{\mathrm{h}, \mathrm{i}}$, Marc Ribo ${ }^{\mathrm{j}}$, Carlos Molina ${ }^{\mathrm{j}}$, Jong-Won Chung ${ }^{\mathrm{k}}$, Oh Young Bang ${ }^{\mathrm{k}}$, Georgios Magoufis ${ }^{1}$, Ahmad Cheema ${ }^{\mathrm{e}}$, Steven J. Shook ${ }^{\mathrm{m}}$, Ken Uchino ${ }^{\mathrm{m}}$, Andrei V. Alexandrov ${ }^{\mathrm{d}}$, Georgios Tsivgoulis ${ }^{\mathrm{b}, \mathrm{d}, *}$

${ }^{a}$ Department of Neurology, Charleston Area Medical Center, West Virginia University-Charleston Division, Charleston, WV, USA

${ }^{\mathrm{b}}$ Second Department of Neurology, National \& Kapodistrian University of Athens, "Attikon" University Hospital, Athens, Greece

${ }^{\mathrm{c}}$ Stroke Unit, Metropolitan Hospital, Piraeus, Greece

${ }^{\mathrm{d}}$ Department of Neurology, University of Tennessee Health Science Center, Memphis, TN, USA

${ }^{\mathrm{e}}$ Department of Neurosurgery, Semmes-Murphey Neurologic and Spine Institute, University of Tennessee Health Science Center, Memphis, TN, USA

${ }^{\mathrm{f}}$ Neurovascular Imaging Research Core and UCLA Stroke Center, Department of Neurology, University of California, Los Angeles, CA, USA

${ }^{\mathrm{g}}$ Department of Neurology, University of Ioannina School of Medicine, Ioannina, Greece

${ }^{\mathrm{h}}$ Department of Neurology, Centro Hospitalar e Universitário de Coimbra, Coimbra, Portugal

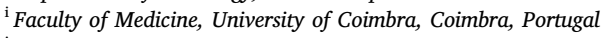

${ }^{\mathrm{j}}$ Vall D'Hebron Stroke Unit, Hospital Universitari Vall D'Hebron Passeig Vall D'Hebron, Barcelona, Spain

${ }^{\mathrm{k}}$ Department of Neurology, Samsung Medical Center, Seoul, South Korea

${ }^{1}$ Department of Interventional Neuroradiology, Metropolitan Hospital, Piraeus, Greece

${ }^{\mathrm{m}}$ Department of Neurology, Cleveland Clinic Foundation, Cleveland, $\mathrm{OH}, \mathrm{USA}$

\section{H I G H L I G H T S}

- Collateral circulation status and final infarct volume (FIV) are independent outcome predictors after an AIS.

- We evaluated the association of statin pretreatment (SP) on collateral circulation and FIV in AIS patients.

- SP was associated with lower FIV compared to no SP, especially in atherosclerotic AIS.

- A trend towards good collateral scores was observed in the SP group.

- SP appears to be associated with decreased FIV after AIS, possibly due to more favorable collateral circulation.

\section{A B S T R A C T}

Background and aims: Statin pretreatment (SP) is associated with improved outcomes in acute ischemic stroke (AIS) patients. Collateral circulation status and final infarct volume (FIV) are independent predictors of functional outcome in AIS.

Methods: We sought to evaluate the association of SP with collateral circulation and FIV in AIS patients. We used a random-effects model for all the analyses, and pooled standardized mean differences (SMDs) and odds ratios (OR) on the FIV and collateral status according to SP history, respectively.

Results: We identified 9 eligible studies (1186 AIS patients). History of SP was associated with lower FIV (SMD $=0.25,95 \%$ CI: $0.07-0.42, p=0.005$ ) compared to negative history of SP. A trend towards good collateral scores was observed in the SP group (OR $=1.45$; 95\% CI, 0.92-2.29, $p=0.11$ ). Subgroup analysis demonstrated reduced FIV among atherosclerotic stroke patients with history of SP (SMD $=0.49 ; 95 \%$ CI, 0.19-0.80, $p=0.001$ ).

Conclusions: SP appears to be associated with decreased FIV, especially in atherosclerotic AIS.

\section{Introduction}

Statin pretreatment (SP) is associated with favorable clinical outcomes in acute ischemic stroke (AIS) due to large artery atherosclerosis (LAA) [1]. Pleiotropic properties of statins extend beyond lipid lowering effects, encompassing positive influence on endothelial

\footnotetext{
${ }^{*}$ Corresponding author. Second Department of Neurology, University of Athens, School of Medicine, Athens, Greece, Iras 39, Gerakas Attikis, Athens, 15344, Greece.

E-mail address: tsivgoulisgiorg@yahoo.gr (G. Tsivgoulis).
} 


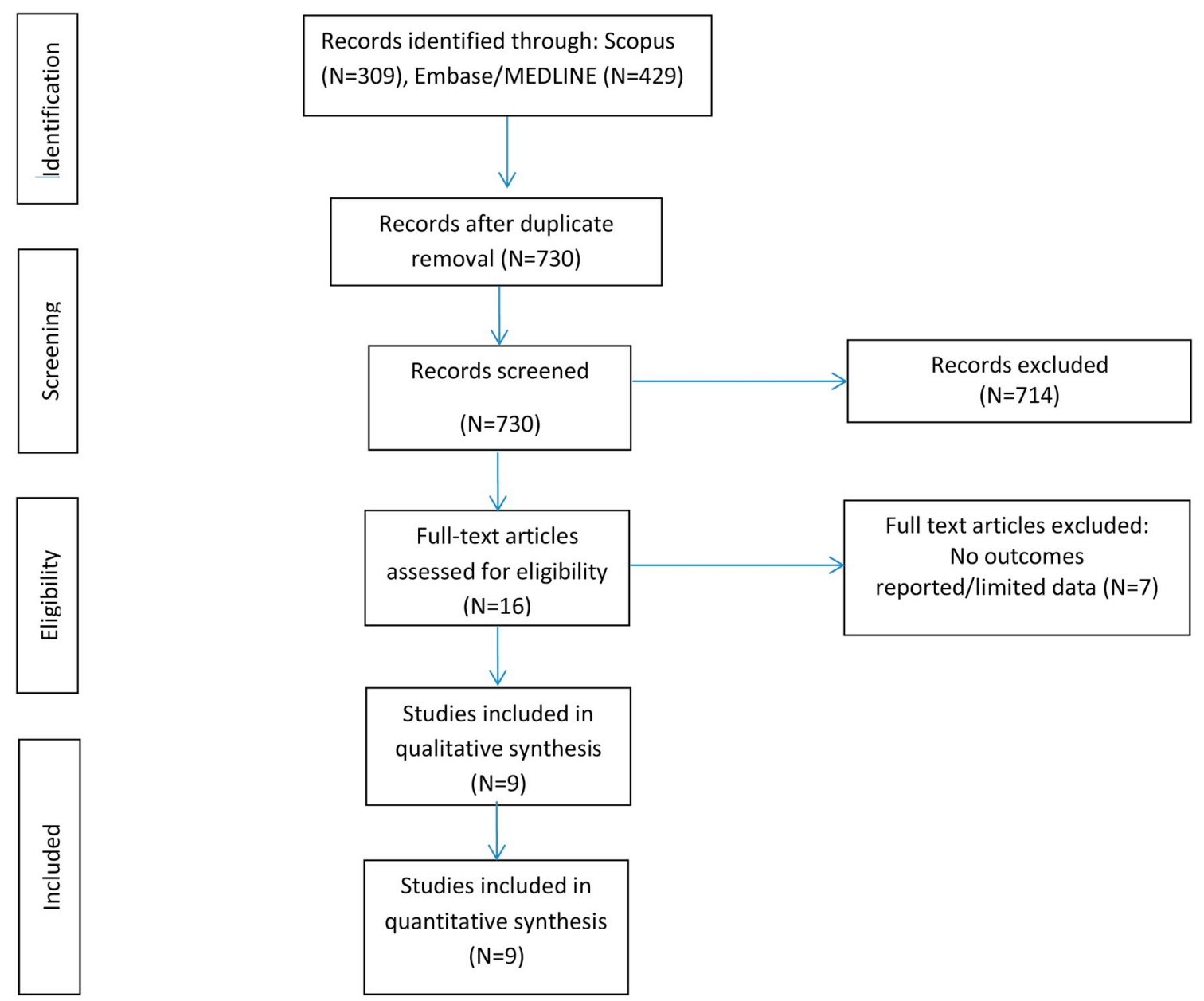

Fig. 1. Flow-chart diagram presenting the selection of eligible studies.

function, cerebral hemodynamics, inflammatory response, platelet function and lipid oxidation [2]. These tissue-level effects further correspond to atherosclerotic plaque stabilization and reduction in stroke recurrence [3]. There are preliminary data showing that SP may be correlated with improved collateralization among AIS patients [4,5]. Moreover, SP have been demonstrated to up-regulate endothelial nitric oxide synthase (eNOS) activity that further enhances collateral perfusion and reduces final infarct volumes (FIV) [6]. Although improved collateral circulation results in reduction of FIV and better clinical outcomes, the potential influence of SP on collateral circulation status and final infarct size has not been determined. In view of these considerations, we performed a systematic review and meta-analysis of available studies reporting FIV or collateral grades based on digital subtraction angiography (DSA) in AIS patients with and without SP.

\section{Materials and methods}

We adopted the Preferred Reporting Items for Systematic Reviews and Meta-Analyses (PRISMA) guidelines for systematic reviews and meta-analyses [7]. We used the Newcastle-Ottawa Scale to explore sources of bias amongst the included observational studies as previously described [8].

We identified all eligible observational studies that investigated the association of statin pre-treatment (SP) prior to admission with collateral circulation and core infarct volume. Systematic search was conducted in Embase/MEDLINE and Scopus databases from inception to June 17, 2018. Additional manual search included conference abstracts and bibliographies of candidate studies and recent systematic reviews for a comprehensive literature search. The detailed search algorithm used on MEDLINE search is outlined in Supplementary Materials. No language or other restrictions was imposed. All retrieved studies were independently reviewed by three authors (KM, AS \& GT), with any disagreement resolved by consensus. We excluded case series, case reports, or studies not reporting any information on SP. In those studies where the original publication reported evidence of SP stratification, but did not contain all necessary data, we contacted the corresponding authors by e-mail. The quality control and bias identification were performed independently by 2 reviewers (KM, AHK), and disagreements, if any, were resolved by a third tie-breaking evaluator (GT).

The corresponding odds ratios (OR) for good collaterals and standardized mean differences (SMD) to assess final infarct volumes (FIV) in patients with SP versus patients without SP were calculated. We also conducted subgroup analyses to evaluate association of SP and FIV among patients with different stroke subtypes. We selected those studies that assessed DSA-based collateral grades according to either the American Society of Interventional and Therapeutic Neuroradiology/ Society of Interventional Radiology (ASITN/SIR) or collateral pial circulation (CPC) collateral flow grading classifications. We defined good collaterals as ASITN/SIR grades 3-4 or CPC scores 0-2, as previously described [2,9]. FIV was defined as infarct regions measured using $\mathrm{ABC} / 2$ method on available neuroimaging during hospitalization [2].

In all analyses, SMDs and ORs of individual studies were pooled using the random-effects model (DerSimonian Laird). Heterogeneity between studies was evaluated using Cochran $\mathrm{Q}$ and $\mathrm{I}^{2}$ statistics, with $\mathrm{I}^{2}$ values of $\mathrm{I}^{2}>50 \%$ and $\mathrm{I}^{2}>75 \%$ indicating substantial and considerable heterogeneity, respectively [10]. Publication bias across individual studies was graphically assessed using a funnel plot, while formal 
assessment with Egger's test could not be performed due to the small number of studies ( $<10$ studies). All statistical analyses were conducted using Review Manager (RevMan) Version 5.3 software (Copenhagen: The Nordic Cochrane Centre, The Cochrane Collaboration, 2014).

\section{Results}

The systematic search of Embase/MEDLINE and Scopus databases yielded 429 and 309 results respectively. After removing duplicates, the titles and abstracts of the remaining 730 studies were screened and, from the 16 potentially eligible studies, 7 studies were excluded for lack of data availability on either collateral scores or infarct volume stratified by SP status (Supplementary Table I). In the final evaluation of the literature search results, there was no conflict or disagreement between reviewers and the 9 studies that met the protocol's inclusion criteria were included in the meta-analysis (Fig. 1) [2,5,6,9,11-15]. The corresponding authors from 7 studies $[2,6,9,11,12,14,15]$ were contacted to obtain necessary unpublished data for the quantitative analyses. Data on collateral status were available as ASITN/SIR $[3,5,9,11,13,14]$ or CPC [2] classification, whereas FIV data were available from 6 studies [2,6,11-14].

The patient data and study protocols of the included studies comprising a total of 1186 patients are summarized in Table 1 and Supplementary Table II. The risk of bias in the included studies was considered to be moderate, mainly because of the lack of reporting appropriate adjustments for potential confounders in 4 study protocols (Supplementary Table III).

$\mathrm{SP}$ was associated with reduced FIV on neuroimaging $(\mathrm{SMD}=0.25$; 95\% CI, 0.07-0.42; Fig. 2A). In studies reporting on collateral status, a trend towards good collateral scores was observed in the SP group $(\mathrm{OR}=1.45$; 95\% CI, 0.92-2.29; Fig. 2B). No evidence of heterogeneity was observed for FIV $\left(\mathrm{I}^{2}=12 \%, p\right.$ for Cochran $\mathrm{Q}$ statistic $\left.=0.34\right)$ or good collateral status $\left(\mathrm{I}^{2}=34 \%, p\right.$ for Cochran $\mathrm{Q}$ statistic $\left.=0.17\right)$. Inspection of the funnel plot revealed no evidence of asymmetry (Supplementary Fig. I and II).

A subgroup analysis comparing stroke subtypes revealed that SP was associated with reduced FIV among patients with atherosclerotic strokes (3 studies; SMD = 0.49; 95\% CI, 0.19-0.80; Fig. 2C). No evidence of heterogeneity was observed $\left(\mathrm{I}^{2}=0 \%, p\right.$ for Cochran $\mathrm{Q}$ statistic $=0.51$ ).

\section{Discussion}

To the best of our knowledge, this is the first meta-analysis demonstrating the beneficial effect of SP among AIS patients in regards to reduced infarct volume. Prior studies have demonstrated improved collateral circulation with SP among acute LAA [2] or cardioembolic strokes $[4,5]$. These beneficial effects are likely attributed to the potential for statins to promote arteriogenesis and augment the eNOSmediated vasodilation [6]. Among AIS patients with symptomatic intracranial atherosclerotic plaques, SP has been associated with reduced plaque enhancement, stabilization of atherosclerotic plaques, and lower frequency of large cortical ischemic strokes [12]. Our meta-analysis corroborates these results as SP in AIS patients is associated with reduced FIV, potentially secondary to better collateralization. Additionally, our subgroup analysis demonstrated a favorable effect of SP on FIV among patients with atherosclerotic strokes.

Our study has limitations. First, eligible studies included observational, small-sized cohorts that could have introduced selection bias. Second, data on both the endpoints (FIV and collateral scores) were unavailable in the majority of included studies. Third, statin type and dosage were reported in only 3 of the included studies (Table 1), while the duration of statin pretreatment was not reported in any of the included studies. Therefore, we were not able to perform further analyses on the duration of pretreatment, dosage or intensity of SP with the outcomes of interest. Moreover, in the present meta-analysis, we were unable to control for potential confounders of the collateral status, including metabolic syndrome, hyperuricemia, age and time from stroke onset to presentation $[16,17]$. Fourth, the evaluation of publication bias using funnel plot inspection was weak, and the possibility of the presence of a small study effect cannot be excluded.

In conclusion, the present meta-analysis adds to the increasing evidence of the beneficial effect of SP in AIS outcomes. SP is associated with reduced infarct volumes, especially in AIS due to LAA. Future prospective observational studies are warranted to confirm these preliminary observations.

\section{Conflicts of interest}

The authors declared they do not have anything to disclose regarding conflict of interest with respect to this manuscript.

Table 1

Study design and characteristics of included studies in our meta-analysis.

\begin{tabular}{|c|c|c|c|c|c|c|c|}
\hline Study name & Country & Study design, registry & Patients, N & Endpoints & $\mathrm{SP}+, \mathrm{SP}-$ & Imaging method & Timing from Index Event \\
\hline \multirow[t]{2}{*}{ Cheng-Ching et al. [14] } & \multirow[t]{2}{*}{ United States } & \multirow[t]{2}{*}{ Retrospective } & \multirow[t]{2}{*}{91} & FIV & 32,58 & DWI-MRI & $<6 h$ \\
\hline & & & & Collaterals (ASITN/SIR) & 20,34 & DSA & - \\
\hline Chung et al. [12] & South Korea & Prospective, STAMINA-MRI & 136 & FIV & $38^{a}, 98$ & DWI-MRI & $<7$ days \\
\hline \multirow[t]{2}{*}{ Goyal et al. [11] } & \multirow[t]{2}{*}{ United States } & \multirow[t]{2}{*}{ Retrospective } & \multirow[t]{2}{*}{116} & FIV & 53,53 & FLAIR-MRI & $<14$ days \\
\hline & & & & Collaterals (ASITN/SIR) & 56,73 & DSA & - \\
\hline Lee et al. [5] & International & Retrospective & 98 & Collaterals & $22^{\mathrm{b}}, 76$ & DSA & $<8 \mathrm{~h}$ \\
\hline Liebeskind et al. [9] & International & Prospective, IMS III & 331 & Collaterals (ASITN/SIR) & 98,178 & DSA & - \\
\hline Safouris et al. [15] & Greece & Prospective & 36 & Collaterals (ASITN/SIR) & 5,31 & DSA & - \\
\hline Sargento-Frietas et al. [2] & Portugal & Retrospective & 118 & Collaterals (CPC) & 17,31 & DSA & - \\
\hline \multirow[t]{2}{*}{ Sheth et al. [13] } & \multirow[t]{2}{*}{ United States } & \multirow[t]{2}{*}{ Retrospective } & \multirow[t]{2}{*}{117} & FIV & 27,89 & DWI-MRI & $<24 \mathrm{~h}$ \\
\hline & & & & Collaterals (ASITN/SIR) & 24,93 & DSA & - \\
\hline Shook et al. [6] & United States & Retrospective & 143 & FIV & $38^{c}, 105$ & DWI-MRI & $<48 \mathrm{~h}$ \\
\hline
\end{tabular}

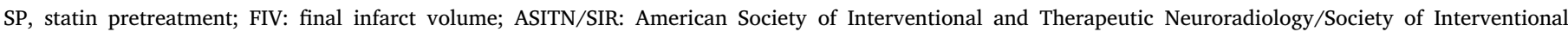

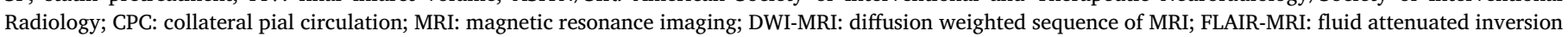
recovery sequence of MRI; DSA: digital subtraction angiography.

a Atorvastatin $<40 \mathrm{mg}$ or equivalent dose $(\mathrm{n}=22)$, $\geq 40 \mathrm{mg}$ atorvastatin or equivalent dose $(\mathrm{n}=16)$.

b Atorvastatin $>10 \mathrm{mg}$ or equivalent $(\mathrm{n}=14)$, atorvastatin $\leq 10 \mathrm{mg}$ or equivalent $(\mathrm{n}=8)$.

c Atorvastatin $(n=24$, median dose $20 \mathrm{mg})$, simvastatin $(\mathrm{n}=10$, median dose $20 \mathrm{mg})$, pravastatin $(\mathrm{n}=3$, median dose $40 \mathrm{mg}$ ), and lovastatin ( $=1$, dose $20 \mathrm{mg})$. 
A.

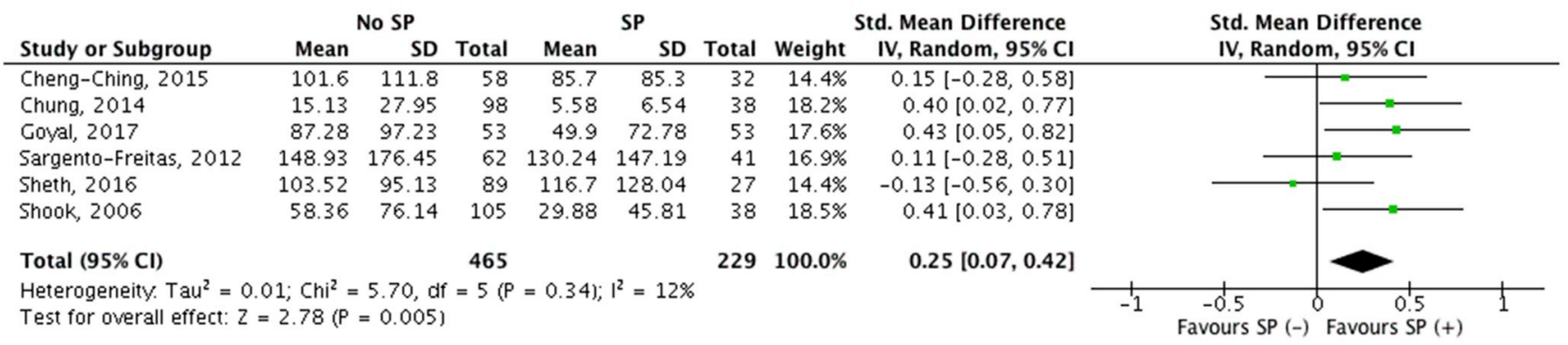

B.

\begin{tabular}{|c|c|c|c|c|c|c|c|c|c|c|}
\hline \multirow[b]{2}{*}{ Study or Subgroup } & \multicolumn{2}{|c|}{ No SP } & \multicolumn{2}{|l|}{ SP } & \multicolumn{2}{|r|}{ Odds Ratio (Non-event) } & \multirow{2}{*}{\multicolumn{3}{|c|}{$\begin{array}{c}\text { Odds Ratio (Non-event) } \\
\mathrm{M}-\mathrm{H} \text {, Random, } 95 \% \mathrm{Cl}\end{array}$}} & \\
\hline & Events & Total & Events & Total & Weight & M-H, Random, 95\% Cl & & & & \\
\hline Liebeskind, 2014 & 66 & 178 & 30 & 98 & $28.3 \%$ & $0.75[0.44,1.27]$ & & $\longrightarrow$ & 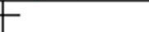 & \\
\hline Cheng-Ching, 2015 & 4 & 34 & 2 & 20 & $5.7 \%$ & $0.83[0.14,5.02]$ & & & & \\
\hline Sheth, 2016 & 39 & 93 & 12 & 24 & $16.4 \%$ & $1.38[0.56,3.41]$ & & & & \\
\hline Goyal, 2017 & 23 & 60 & 32 & 56 & $20.6 \%$ & $2.14[1.02,4.51]$ & & & & \\
\hline Sargento-Freitas, 2012 & 9 & 17 & 22 & 31 & $10.6 \%$ & $2.17[0.64,7.42]$ & & & & \\
\hline Safouris, 2018 & 20 & 31 & 4 & 5 & $3.6 \%$ & $2.20[0.22,22.20]$ & & & & \\
\hline Lee, 2014 & 21 & 76 & 11 & 22 & $14.8 \%$ & $2.62[0.99,6.95]$ & & & & \\
\hline Total $(95 \% \mathrm{Cl})$ & & 489 & & 256 & $100.0 \%$ & $1.45[0.92,2.29]$ & & & & \\
\hline Total events & 182 & & 113 & & & & & & & \\
\hline $\begin{array}{l}\text { Heterogeneity. } \mathrm{Tau}^{2}=0 \\
\text { Test for overall effect: } z\end{array}$ & $\begin{array}{l}\text { 12; } \mathrm{Chi}^{2} \\
1.59(\mathrm{P}\end{array}$ & $\begin{array}{l}=9.08, \\
=0.11\end{array}$ & df $=6($ & $P=0$. & $7) ; 1^{2}=$ & & 0.05 & $\begin{array}{c}0.2 \\
\text { Favours SP (-) }\end{array}$ & 1 Favours SP & 20 \\
\hline
\end{tabular}

C.

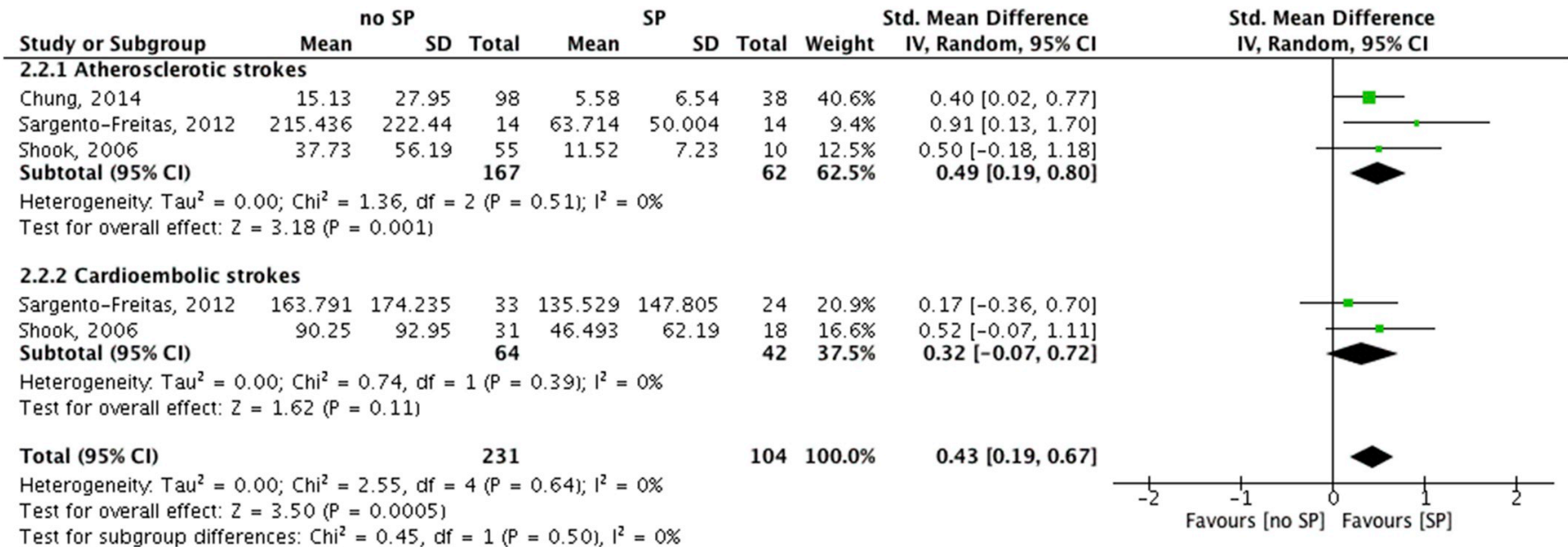

Fig. 2. Forest plots.

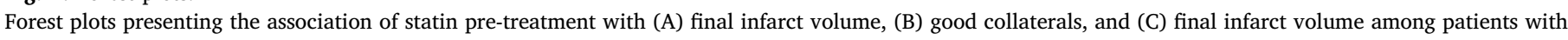
atherosclerotic or cardioembolic stroke subtypes as subgroup analyses.

\section{Appendix A. Supplementary data}

Supplementary data to this article can be found online at https:// doi.org/10.1016/j.atherosclerosis.2019.01.006.

\section{References}

[1] G. Tsivgoulis, A.H. Katsanos, V.K. Sharma, C. Krogias, R. Mikulik, K. Vadikolias, et al., Statin pretreatment is associated with better outcomes in large artery atherosclerotic stroke, Neurology 86 (2016) 1103-1111.

[2] J. Sargento-Freitas, J. Pagola, M. Rubiera, A. Flores, F. Silva, D. Rodriguez-Luna, et al., Preferential effect of premorbid statins on atherothrombotic strokes through collateral circulation enhancement, Eur. Neurol. 68 (2012) 171-176.

[3] A. Safouris, A.H. Katsanos, A. Kerasnoudis, C. Krogias, J.A. Kinsella, R. Sztajzel, et al., Statin pretreatment and microembolic signals in large artery atherosclerosis: a systematic review and meta-analysis, Stroke 49 (2018) 1992-1995.

[4] B. Ovbiagele, J.L. Saver, S. Starkman, D. Kim, L.K. Ali, R. Jahan, et al., Statin enhancement of collateralization in acute stroke, Neurology 68 (2007) 2129-2131.

[5] M.J. Lee, O.Y. Bang, S.J. Kim, G.M. Kim, C.S. Chung, K.H. Lee, et al., Role of statin in atrial fibrillation-related stroke: an angiographic study for collateral flow, Cerebrovasc. Dis. (Basel, Switzerland) 37 (2014) 77-84.

[6] S.J. Shook, R. Gupta, N.A. Vora, A.L. Tievsky, I. Katzan, D.W. Krieger, Statin use is independently associated with smaller infarct volume in nonlacunar mca territory stroke, J. Neuroimaging: Off. J. Am. Soc. Neuroimaging 16 (2006) 341-346.

[7] A. Liberati, D.G. Altman, J. Tetzlaff, C. Mulrow, P.C. Gotzsche, J.P. Ioannidis, et al., The prisma statement for reporting systematic reviews and meta-analyses of studies that evaluate health care interventions: explanation and elaboration, J. Clin. Epidemiol. 62 (2009) e1-34.

[8] A.H. Katsanos, J. Parissis, A. Frogoudaki, A.R. Vrettou, I. Ikonomidis, I. Paraskevaidis, et al., Heart failure and the risk of ischemic stroke recurrence: a systematic review and meta-analysis, J. Neurol. Sci. 362 (2016) 182-187.

[9] D.S. Liebeskind, T.A. Tomsick, L.D. Foster, S.D. Yeatts, J. Carrozzella, 
A.M. Demchuk, et al., Collaterals at angiography and outcomes in the interventional management of stroke (ims) iii trial, Stroke 45 (2014) 759-764.

[10] J.P. Higgins, S. Green, Cochrane Handbook for Systematic Reviews of Interventions, (2005).

[11] N. Goyal, G. Tsivgoulis, S. Iftikhar, Y. Khorchid, M. Fawad Ishfaq, V.T. Doss, et al., Admission systolic blood pressure and outcomes in large vessel occlusion strokes treated with endovascular treatment, J. Neurointerventional Surg. 9 (2017) 451-454.

[12] J.W. Chung, J. Hwang, M.J. Lee, J. Cha, O.Y. Bang, Previous statin use and highresolution magnetic resonance imaging characteristics of intracranial atherosclerotic plaque: the intensive statin treatment in acute ischemic stroke patients with intracranial atherosclerosis study, Stroke 47 (2016) 1789-1796.

[13] S.A. Sheth, N. Sanossian, Q. Hao, S. Starkman, L.K. Ali, D. Kim, et al., Collateral flow as causative of good outcomes in endovascular stroke therapy, J.
Neurointerventional Surg. 8 (2016) 2-7.

[14] E. Cheng-Ching, J.A. Frontera, S. Man, J. Aoki, Y. Tateishi, F.K. Hui, et al., Degree of collaterals and not time is the determining factor of core infarct volume within 6 hours of stroke onset, AJNR. Am. J. Neuroradiol. 36 (2015) 1272-1276.

[15] A. Safouris, O. Kargiotis, K. Psychogios, S. Spiliopoulos, K. Palialexis, et al., Baseline collateral circulation is associated with early outcomes in patients with large-vessel occlusion acute ischemic stroke treated with mechanical thrombectomy, Nevrologia 27 (2018) 6-13.

[16] B.K. Menon, E.E. Smith, S.B. Coutts, et al., Leptomeningeal collaterals are associated with modifiable metabolic risk factors, Ann. Neurol. 74 (2013) 241-248.

[17] D.S. Liebeskind, R. Jahan, R.G. Nogueira, et al., Early arrival at the emergency department is associated with better collaterals, smaller established infarcts and better clinical outcomes with endovascular stroke therapy: SWIFT study, J. Neurointerventional Surg. 8 (2016) 553-558. 